\title{
Guidelines for the Management of Center-Involving Diabetic Macular Edema: Treatment Options and Patient Monitorization
}

João Figueira, (iD ${ }^{1,2}$ José Henriques, ${ }^{3}$ Ângela Carneiro, ${ }^{4,5}$ Carlos MarquesNeves, ${ }^{6-8}$ Rita Flores, ${ }^{9,10}$

João Paulo Castro-Sousa, ${ }^{11-13}$

Angelina Meireles, (ID) ${ }^{14,15}$

Nuno Gomes, ${ }^{16}$ João Nascimento, ${ }^{17}$

Miguel Amaro, ${ }^{18}$ Rufino Silva (D) 1,2,19

'Ophthalmology Department, Centro

Hospitalar e Universitário de Coimbra (CHUC),

Coimbra, Portugal; Faculty of Medicine,

University of Coimbra (FMUC), Coimbra,

Portugal; ${ }^{2}$ AIBILI - Association for Innovation and

Biomedical Research on Light and Image,

Coimbra, Portugal; ${ }^{3}$ Retina Department, Retinal

Surgical Unit, Dr. Gama Pinto Ophthalmology

Institute, Lisbon, Portugal; ${ }^{4}$ Department of

Ophthalmology, Centro Hospitalar Universitário

de São João, Porto, Portugal; ${ }^{5}$ Department of

Surgery and Physiology, Faculty of Medicine of

University of Porto, Porto, Portugal;

${ }^{6}$ Department of Ophthalmology, Centro

Hospitalar Universitário de Lisboa Norte, EPE -

Hospital de Santa Maria, Lisbon, Portugal;

${ }^{7}$ Department of Ophthalmology, Faculdade de

Medicina, Universidade de Lisboa, Lisbon,

Portugal; ${ }^{8}$ ALM Oftalmolaser, Lisbon, Portugal;

${ }^{9}$ Department of Ophthalmology, Centro

Hospitalar de Lisboa Central EPE, Lisbon,

Portugal; ${ }^{10} \mathrm{CEDOC}$, Chronic Diseases Research

Center, NOVA Medical School, Lisbon, Portugal;

"Department of Ophthalmology, Centro

Hospitalar de Leiria, Leiria, Portugal; ${ }^{12} \mathrm{CICS}$-UBI,

Health Sciences Research Centre, University of

Beira Interior, Covilhã, Portugal; ${ }^{13}$ Faculty of

Medical Sciences, Universidade da Beira Interior,

Covilhã, Portugal; ${ }^{14}$ Ophthalmology

Department, Centro Hospitalar Universitário do

Porto, Porto, Portugal; ${ }^{15}$ Instituto de Ciências

Biomédicas Abel Salazar, Universidade do Porto, Porto, Portugal; ${ }^{16}$ Ophthalmology Department, Hospital de Braga, Braga, Portugal; ${ }^{17}$ Instituto de Retina e Diabetes Oculares de Lisboa, Lisbon, Portugal; ${ }^{18}$ Ophthalmology Department, Hospital Vila Franca de Xira, Vila Franca de Xira, Portugal; ${ }^{19}$ Coimbra Medical Space, Coimbra, Portugal

Correspondence: João Figueira

Praceta Mota Pinto, Coimbra, 3000-075,

Portugal

$\mathrm{Tel}+351239701182$

Email joaofigueira@oftalmologia.co.pt

\begin{abstract}
Diabetic macular edema (DME) is the main cause of visual impairment associated with diabetic retinopathy (DR) and macular laser, during approximately three decades, and was the single treatment option. More recently, intravitreous injections of anti-angiogenics and corticosteroids modified the treatment paradigm associated with significant vision improvements. Nevertheless, not all patients respond satisfactorily to anti-VEGF or corticosteroid injections, so an adequate treatment choice and a prompt switch in therapeutic class is recommended. Several algorithms and guidelines have been proposed for treating center involving DME to improve patients' vision and quality of life. However, in Portugal, such guidelines are lacking. The present review aimed to provide guidelines for the treatment options and patient monitorization in the management of center-involving DME. We recommend anti-vascular endothelial growth factor (VEGF) as first-line therapy after a clinical evaluation accompanied by a rigorous metabolic control. Depending on the response obtained after 3-6 monthly intravitreal injections we suggest switching outside the class in case of a non-responder, maintaining the anti-VEGF-therapy in responders to anti-angiogenics. The treatment regimen for Dexamethasone intravitreal implant (DEXii) should be pro-re-nata with bi-monthly or quarterly monitoring visits (with a scheduled visit at 6-8 weeks after DEXii for intraocular pressure control). If a patient does not respond to DEXii, switch again to anti-VEGF therapy, combine therapies, or re-evaluate patients diagnose. There is a resilient need to understand the disease, its treatments, regimens available, and convenience for all involved to propose an adequate algorithm for the treatment of diabetic retinopathy (DR) and DME in an individualized regimen. Further understanding of the contributing factors to the development and progression of DR should bring new drug discoveries for more effective and better-tolerated treatments.
\end{abstract}

Keywords: anti-vascular endothelial growth factor, center-involving diabetic macular edema, dexamethasone intravitreal implant, diabetic retinopathy

\section{Introduction}

Diabetic macular edema (DME) commonly leads to impairment of visual acuity in patients with non-proliferative diabetic retinopathy (NPDR) and is more prevalent in patients with type 2 diabetes mellitus (DM2) than type 1 (DM1), accounting to nearly $12.9 \%$ and $7.86 \%$ cases, respectively. ${ }^{1}$ The causes of DME are multifactorial; however, the predominant reason is attributable to blood-retinal barrier breakdown that leads to swelling in the Henle's layer of the macula. ${ }^{2}$

Diabetic retinopathy (DR) and DME are currently treated with a rigorous systemic and ocular management. ${ }^{2}$ Controlling glucose, blood pressure, and blood 
lipids, as well as other multifactorial interventions, are important elements of systemic treatment. ${ }^{3}$ One of the goals for successful management of diabetes and its consequences is to maintain HbA1c levels between $6 \%$ and $7 \%$. Because a high blood glucose level is the most modifiable risk factor, educating the patient to a healthy lifestyle with good control of their disease is important for blood glucose levels, blood pressure, and serum lipid levels. ${ }^{3,4}$ The primary care doctor, nurse, endocrinologist, and ophthalmologist should all be actively involved in this control and monitoring.

In terms of ocular treatment, there are presently a variety of pharmacological and non-drug options available, albeit none of them cure the diseases, but only ameliorate symptoms and delay disease progression. ${ }^{3}$ The most current and available treatment options are laser photocoagulation, pharmacological agents injected in the vitreous body, namely anti-VEGF and corticosteroids, and vitrectomy. $^{2}$

Laser photocoagulation was one of the first noninvasive treatments to have a low rate of complications and a high rate of success. The Early Treatment Diabetic Retinopathy Study (ETDRS) was a landmark clinical trial that demonstrated the efficacy of the focal macular laser in the treatment of diabetic retinopathy and diabetic macular edema (DME). ${ }^{5}$ The criteria for treating "clinically significant macular edema" (CSME) were defined in this study, and they were used to determine which patients should be treated with macular laser. ${ }^{6}$ According to the ETDRS, laser photocoagulation reduced the probability of moderate vision loss by around $50 \%$ and improved the vision of around $30 \%$ of the patients. However, despite photocoagulation treatment, patients still experience vision loss. ${ }^{6}$ To date, there has been a substantial shift in the type of laser treatments from traditional lasers to more advanced technologies, such as micropulses, which have increased the therapeutic benefits of laser use while reducing retinal damage. ${ }^{7}$ Unlike conventional lasers, subthreshold micropulse laser treatment does not damage neurosensorial retinal cells. The importance of laser photocoagulation complications has lessened with the development of newer lasers, but the principle of thermal destruction of retinal cells has stayed unchanged. Several investigations have shown that subthreshold micropulse laser treatment is safe and does not damage the retinal pigment epithelium or photoreceptors. 8,9

It should be noted, therefore, that the morphological improvement is greater than the functional improvement and because of this, pharmacological treatments such as anti-VEGF and corticosteroids are often considered for DME, with functional benefits superior to subthreshold micropulse laser treatment. ${ }^{10,11}$ According to Euretina guidelines, laser photocoagulation is not recommended for the treatment of DME. ${ }^{4}$ Nevertheless, in certain circumstances, subthreshold micropulse laser treatment may be considered when other therapies are unavailable or contraindicated, such as during pregnancy or breastfeeding, or when other treatments are ineffective.

The development of new treatment options and diagnostics tools has significantly improved the management of DME.

The pharmacological treatments primarily include antivascular endothelial growth factor (VEGF) medications and corticosteroids. The former includes aflibercept (Eylea $^{\circledR}$; Regeneron Pharmaceuticals Inc., Tarrytown, NY, USA, and Bayer HealthCare Pharmaceuticals, Berlin, Germany), ranibizumab (Lucentis ${ }^{\circledR}$; Genentech Inc., South San Francisco, CA, USA), and bevacizumab (Avastin $^{\circledR}$; Genentech, South San Francisco, CA, USA/ Roche, Basel, Switzerland), which is an off-label therapy. ${ }^{12}$ VEGF inhibition has been widely used and has established itself as the gold standard for the treatment of DR associated with DME. ${ }^{12}$

Aflibercept, also known as VEGF-Trap, is a fusion protein that combines the ligand-binding components of VEGFR-1 and VEGFR-2 extracellular domains with the Fc part of IgG. This anti-angiogenic inhibits tumor growth and vascularization. ${ }^{13}$ Furthermore, this anti-VEGF binds to all VEGF-A, VEGF-B, and PIGF isoforms. ${ }^{13}$

Ranibizumab is a recombinant humanized monoclonal antibody fragment (Fab) generated in an Escherichia coli production system (and thus not glycosylated). It was genetically modified to maximize its affinity for binding and inhibition of VEGF-A, and it was specially developed to be used intravitreally, having been licensed by the FDA and EMA for the treatment of several retinal disorders. ${ }^{14}$

Bevacizumab is approved for the treatment of patients with metastatic colon, rectum, or breast cancer, as well as individuals with non-small cell lung cancer or metastatic renal cell carcinoma. However, it is utilized off-label in ophthalmology. It is a full-length recombinant humanized monoclonal antibody with both Fc and Fab regions that is three times larger than ranibizumab being generated in the mammalian expression system (glycosylated molecule). The Fc antibody domain contributes to immune activation. $^{14}$ 
In the DRCRnet clinical trials, specifically in the $\mathrm{T}$ protocol, which compared the 3 anti-angiogenic drugs, Eylea ${ }^{\circledR}$ (aflibercept), Lucentis ${ }^{\circledR}$ (ranibizumab), and Avastin $^{\circledR}$ (bevacizumab) in the treatment of DME, patients with visual acuity of $20 / 50$ or worse at the start of the study had superior outcomes with aflibercept at the end of the first year. However, there were no differences in results between the three drugs at the end of the first year in patients with baseline vision of 20/40 to 20/ 32. At the end of protocol $\mathrm{T}$ second year, the only statistical difference in acuity among the three drugs was aflibercept's superiority to bevacizumab in eyes with 20/50 or worse baseline vision. Furthermore, there were no significant differences regarding drug safety between bevacizumab, ranibizumab, and aflibercept in the treatment of DME. Notwithstanding, not all patients respond sufficiently to anti-VEGF therapy, prompting clinicians to switch to other available therapies or novel research approaches. ${ }^{15}$

The latter include Dexamethasone intravitreal implant (DEXii), (Ozurdex ${ }^{\circledR}$; Allergan Inc., Irvine, CA), an injectable fluocinolone polymer (FAc), (Iluvien ${ }^{\mathbb{B}}$; Alimera Sciences, Alpharetta, GA), and triamcinolone acetonide (TA) (an off-label therapy). ${ }^{16}$

Corticosteroids have a significant role in the treatment of DME. Despite the many benefits of intravitreous steroid therapy, this treatment is associated with the risk of adverse events such as increased intraocular pressure and cataract formation. ${ }^{16}$ The role of corticosteroids in the treatment of DME is multifactorial. Corticosteroids are potent anti-inflammatory drugs that also antagonize VEGF-A function, inhibit leukostasis, and reduce inflammatory cytokines. ${ }^{17}$ DEXii was created to ensure a continuous release of the medication into the vitreous for approximately 4 to 6 months. $^{18}$ DEXii is a biodegradable implant that contains $0.7 \mathrm{mg}$ DEX and is made of polyglycolic acid and polylactic acid polymers and has been shown to be effective in treating a variety of retinal disorders, including $\mathrm{ME}, \mathrm{DME}$, and retinal vein occlusion. ${ }^{18}$ FAc is a nonbiodegradable implant that contains $0.19 \mathrm{mg}$ of FAc and is meant to deliver $0.20 \mu \mathrm{g}$ of FAc per day for three years. ${ }^{19}$

As corticosteroids trigger cataract development in phakic eyes, the visual results may be distorted by cataract progression. Nevertheless, according to a subgroup analysis of pseudophakic eyes in Protocol I of the Diabetic Retinopathy Clinical Research (DRCR) network at two years, the results of the triamcinolone acetonide arm were equivalent to the ranibizumab arms. ${ }^{20}$ IOP is another side effect of corticosteroids; however, it is usually treatable with intraocular drops. ${ }^{21}$

Despite the well-known adverse events of corticosteroids, such as cataract formation and increased intraocular pressure, efficacy had already shown that the benefits may outweigh in specific cases of the risks. Furthermore, intravitreal corticosteroids provide a significant advantage since the systemic side effects of intra-ocular administered corticosteroids occur rarely. ${ }^{21}$

Several randomized controlled trials (RCTs) have reported the success of DEXii, aflibercept, and ranibizumab, and these are now approved for this indication by the Food and Drug Administration (FDA), European Medicines Agency (EMA), and other regulatory authorities throughout the world. ${ }^{18-23}$ Many protocols, algorithms, clinical trials, and observational studies have resulted in an extreme variety of treatment regimens and patient's follow-up options; however, these are sometimes not well adapted to clinical practice. Furthermore, clear guidelines for Portuguese ophthalmologists to address patients' treatment options and follow-up, besides what is stated in the summary product characteristics, in the realworld studies, in the countries' guidelines and published by international ophthalmology organizations, are limited. Therefore, the present review was aimed to provide guidelines for the treatment options and patient monitorization in the management of center-involving DME.

\section{Materials and Methods Literature Search Strategy}

Literature describing the management of DME was searched from PubMed and Google Scholar. The papers chosen were published between 2010 and 2020. Only peerreviewed publications were included in the selection of papers, which were all written in the English language. The keywords for the search included "management of diabetic macular edema", OR "diabetic retinopathy", OR "anti-vascular endothelial growth factor", OR "Ozurdex", OR "dexamethasone intravitreal implant", OR "Iluvien" OR "fluocinolone acetonide intravitreal implant", in the title or the manuscript text. Only human research was included and remains were excluded. In addition, the references of relevant papers were examined for completeness of our search and also to include new studies, additional important data, and guidelines. 


\section{Data Extraction, Quality Assessment, and Endpoints}

The titles and abstracts were examined by two researchers independently, and full articles were chosen for inclusion.

All authors read and analysed the articles selected for inclusion in the article, and they were judged to be appropriate for inclusion in this manuscript based on the major objectives or endpoint of this recommendation. Disagreements were settled by discussion and agreement. Moreover, the findings of all the relevant articles were collected, comprehensively analysed, and discussed by a group of retinal experts.

\section{Data Analysis}

Information retrieved was analysed and discussed equally by all authors, during consensus meetings. No statistical analysis was carried out for this guideline.

\section{Discussion}

\section{Treatment Options}

The intravitreal levels of VEGF are reportedly higher in patients with diabetes. ${ }^{24}$ Therefore, intravitreal anti-VEGF medications, such as ranibizumab, aflibercept, and bevacizumab are usually the first line of treatment for DME. AntiVEGF-based treatment regimen is continued if the patient responds well; however, the strategy is moved to intravitreal corticosteroids, normally DEXii when the patient does not respond to anti-VEGF-based treatment even after 3-6 monthly intravitreal injections. DEXii is also preferred for patients not suitable for first-line VEGF therapy or those who are unavailable for frequent check-ups, while FAc has been used for more chronic and resistant DME or when a very long steroid action is desired. The pro-re-nata $(\mathrm{PRN})$ treatment regimen should include bimonthly or quarterly eye check-ups and intraocular pressure control (IOP) control in patients at 2 months after each DEXii. In cases where steroid treatment is not effective, the course should involve switching again to anti-VEGF-based strategy with or without combination therapy, including laser with ETDRS protocol 2007 or more retina-friendly laser parameters and if needed, re-evaluation of the diagnostic can be performed. $^{25,26}$

\section{Factors Affecting Treatment}

Patients with diabetes having DME or proliferative diabetic retinopathy (PDR) have higher risk of cardiovascular diseases, and arteriothrombotic events, such as stroke or myocardial infarction. ${ }^{27}$ Therefore, evaluation of patients' medical history, including diabetes status, cardiovascular events, and other comorbidities, is imperative. Anti-VEGF are not forbidden in these cases. However, the risk-benefit should be discussed and evaluated case by case.

\section{Ophthalmologic Factors}

Proliferative Diabetic Retinopathy

Anti-VEGF agents, aflibercept and ranibizumab and the off-label use of bevacizumab, should be the 1st line treatment option in eyes with DME associated with PDR, as suggested by the regression in neovascularization in the RISE and RIDE, RESOLVE, RESTORE and RETAIN, VIVID and VISTA. ${ }^{28}$

\section{Vitrectomized Eyes}

DEXii can be used as 1st line therapy in vitrectomized patients. These have been found useful, especially in treating inflammation and $\mathrm{ME}$ in difficult-to-treat vitrectomized eyes, showing vascular leakage and best corrected visual acuity (BCVA) improvement. ${ }^{29,30}$

\section{Tractional Edema}

Based on the literature and real-world experience we can recommend PPV plus DEXii as first-line therapy when traction is present. It has shown improvements in BCVA, central retinal thickness and macular volume with no apparent hemorrhage or trauma to the retina, as well as decreased macular edema and improved visual outcome after macular epiretinal membrane removal. ${ }^{31,32}$

\section{Intraocular Pressure}

We recommend following the proposed algorithm in case of controlled IOP (Figure 1). However, in case of uncontrolled IOP, the 1st line treatment should include anti-VEGF and DEXii should be considered after discussion of the riskbenefit. Anti-VEGF is preferable in patients with uncontrolled or severe glaucoma (double or triple combination therapy). Conversely, DEXii and FAc are acceptable in patients with no glaucoma or glaucoma treated with monotherapy. ${ }^{21,33,34}$

Nevertheless, after DEXii patients should perform a safety visit after 6-8 weeks of implantation to evaluate the therapeutic response to corticosteroids and any potential increase in $\mathrm{IOP}^{33}$

\section{Inflammation Biomarkers}

Several inflammatory retinal biomarkers have been proposed to identify DME cases where the inflammatory pathway is prominent. $^{35}$ The image biomarkers mostly studied include disorganization of retinal inner layers (DRIL), subretinal fluid (SRF), hyperreflective retinal spots (HRS), hard exudates (Figure 2). ${ }^{36-39}$ 


\section{Proposed Algorithm For The Treatment Of CiDME}

\section{Clinical Evaluation + Metabolic Control}
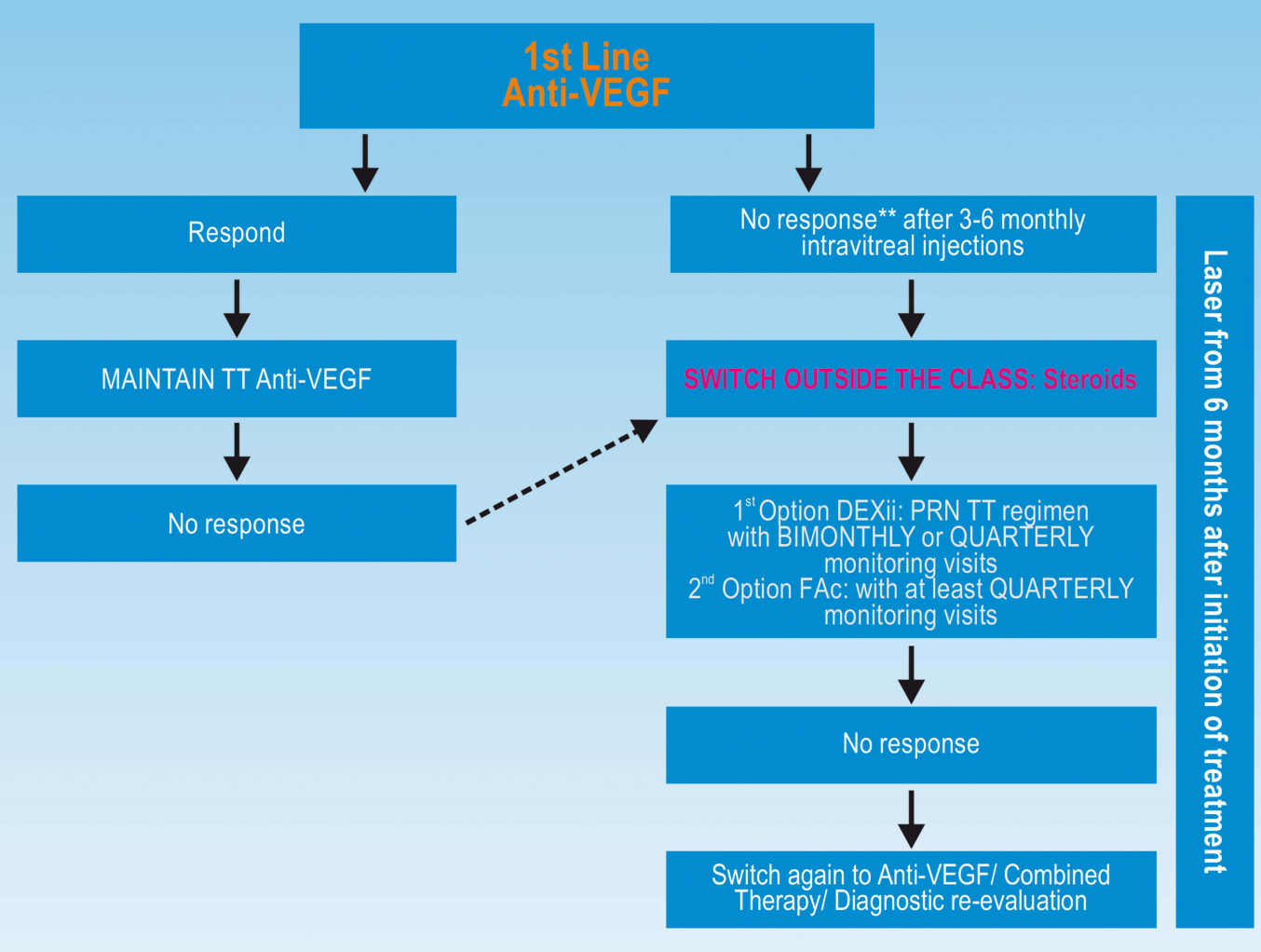

Clinical Evaluation + Metabolic Control - Other factors to be considered

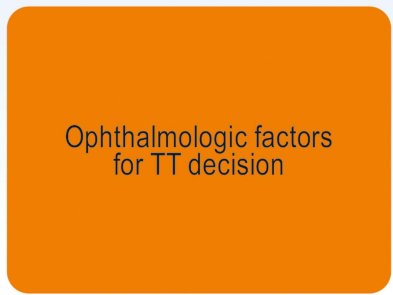

1 PDR: $1^{\text {st }}$ Line Anti-VEGF

2 Vitrectomized: $1^{\text {st }}$ Line DEXii

3 Ischemia: Anti-VEGF/ DEXii

4 Inflammation biomarkers: Probably steroids (additional studies are needed)

5 Tractional edema: $1^{\text {st }}$ Line Vitrectomy + DEXii

6 IOP: a. If controlled IOP: follow proposed algorithm; b. If uncontrolled IOP: $1^{\text {st }}$ Line Anti-VEGF or discuss risk-benefit of steroids

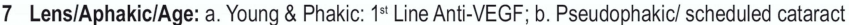
surgery: $1^{\text {st }}$ Line Steroids/Anti-VEGF; c. Aphakic/ without capsular or zonular integrity: $1^{\text {st }}$ Line Anti-VEGF

Systemic factors for TT decision

1 Recent CVD/ recent ATEs: $1^{\text {st }}$ Line DEXii/Anti-VEGF (discuss risk-benefits)

2 Pregnancy / breastfeeding: Laser and/or DEXii

Other factors for TT decision: Compliance

$11^{\text {st }}$ Line DEXii

Figure I Proposed algorithm for the treatment of CiDME.

Abbreviations: Anti-VEGF, anti-vascular endothelial growth factor; ATEs, arterial thromboembolic events; CiDME, center involved DME; CMT, center macular thickness; CVC, cardiovascular diseases; DEXii - dexamethasone intravitreal implant; FAc, fluocinolone acetonide intravitreal implant; IOP, intraocular pressure; NVC, neovascularization; PRN, pro-re-nata or as needed; TT, treatment. 


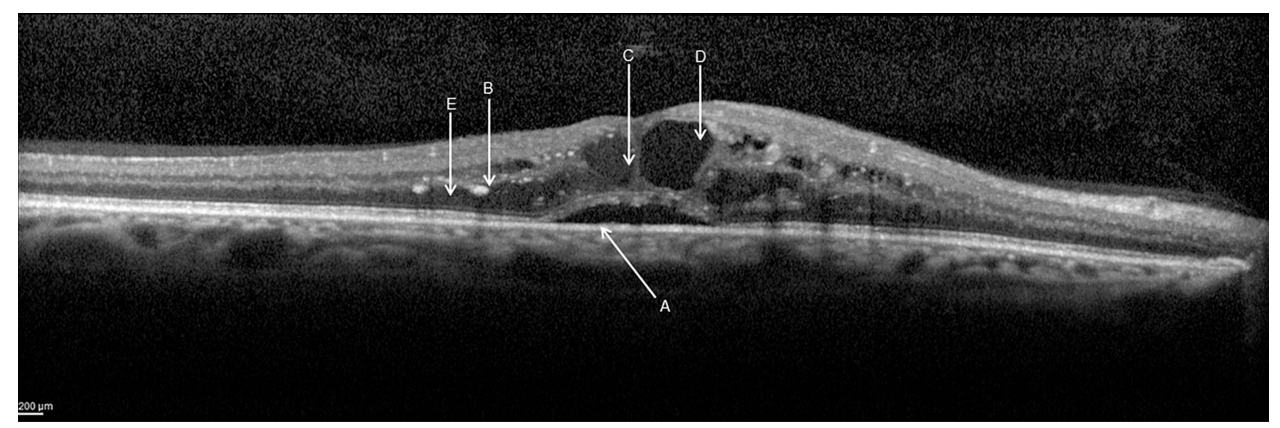

Figure 2 OCT biomarkers in DME: A. Subretinal fluid (SRF), B. Hyperreflective retinal spots (HRS), C. Intraretinal cysts (IRC), D. Disorganization of retinal inner layers (DRIL), E. Hard exudates. Courtesy Ana Rita Santos.

Meduri et al investigated the safety and efficacy of DEXii as first-line therapy in DME-naïve patients, demonstrating the importance of OCT biomarkers as predictors of response. The existence of SRD, the integrity of EZ (ellipsoid zone), and the lack of vitreomacular abnormalities were predictors of a positive response to DEXii. ${ }^{40}$

Probably, intravitreous corticosteroids can be a good treatment option in the presence of such biomarkers, but additional studies are needed to confirm this theory.

\section{Lens Status and Age}

Lens status should be evaluated before patients' treatment with any class of corticosteroids. Depending on lens status, aphakic, or age, the recommendation for therapy varies. The studies with corticosteroids intravitreous implants for DME have reported cataract-associated adverse events in phakic eyes and increased vision loss in patients treated with DEX or FAc. ${ }^{41,42}$ Therefore, in a young and phakic patient, we recommend first-line anti-VEGF treatment; however, in a pseudophakic patient or a patient with a scheduled cataract surgery DEXii as first-line treatment can be considered. For aphakic patients or those without capsular or zonular integrity, we recommend anti-VEGF as first-line treatment due to the risk of the corticosteroids implant migration to the anterior chamber. ${ }^{43}$

\section{Ischemia}

In cases of DME with macular ischemia associated, the therapeutic options remain controversial. Multicenter studies confirmed the efficacy of anti-VEGFs in DME however, have excluded eyes with macular ischemia, so it is not possible to conclude about the efficacy of these drugs in these specific cases. Although the ETDRS study recommends laser photocoagulation in those cases, the visual prognosis is worse than without ischemia, and some authors do not even recommend it. ${ }^{44,45}$
Despite the poor prognosis, it is suggested to treat patients with anti-VEGF drugs, monitor the visual acuity and the evolution of the DME with OCT monthly. If there is no improvement in visual acuity after the edema resolution, treatment should be suspended. Intravitreous corticosteroids may be an alternative in cases where anti-VEGFs are contraindicated or when the latter has proven ineffective in reducing DME. As with anti-VEGFs, also in these cases, if the resolution of the DME is not accompanied by recovery of visual acuity, treatment should be discontinued. ${ }^{46}$

\section{Systemic Factors}

\section{Recent Cardiovascular Disease (CVD)/Recent Arteriothromboembolic Events}

There is a proven correlation between diabetes and CVD. ${ }^{47}$ The diabetic population has several risk factors that contribute to the development of CVD, such as hypertension, abnormal cholesterol, and high triglycerides values, obesity, lack of physical activity, smoking habits and lastly poor controlled blood sugar levels. ${ }^{47}$ AntiVEGF agents can potentially increase systemic adverse events such as kidney disease, gastrointestinal perforations, hypertension, stroke, myocardial infarction, and thromboembolic events, due to which a black box has been added in the summary of product characteristics of bevacizumab, ranibizumab, and aflibercept. ${ }^{48,49}$

Based on the evidence published concerning the longterm systemic safety profile of corticosteroids, we recommend DEXii as first-line therapy amid corticosteroids.

\section{Pregnancy/Breastfeeding}

DME treatment during pregnancy may be difficult owing to questions regarding the potential adverse antiangiogenic effects of anti-VEGF therapies on a developing fetus. ${ }^{50}$

If DME is present during the pregnancy, observation is a reasonable management option for pregnant patients 
with mild DME, since the edema may well resolve after delivery. For DME requiring treatment, we believe for safety reasons that anti-VEGF therapy should be avoided in favor of focal laser photocoagulation or DEXii. ${ }^{51,52}$

\section{Other Factors for Treatment Decision: Patient Compliance}

Although continuity with treatment is essential for its success, compliance with frequent anti-VEGF injections is a recognized problem with this paradigm regarding recurrent neovascularization and hemorrhage. Evaluating patient's willingness to adhere to health care provider's recommendations is a key factor. ${ }^{53,54}$ The introduction of longer duration-of-action anti-VEGF drugs, currently under development for DME, such as faricimab or brolucizumab, may lower the number of requested injections and follow-up visits, improving patient's compliance.

Therefore, in cases of non-compliance or impossibility due, for example, to socio-economic reasons, to return to treatment or follow-up visits we can recommend DEXii as first-line treatment.

\section{Treatment Regimens and Monitorization}

Anti-VEGFs are normally used in PRN regimen after 3 to 6 monthly intravitreal injections, or in fixed regimens or in a treat and extend (TE) approach. Monitoring, either clinical or imagiological must be adjust to each treatment regimen. ${ }^{34,55}$ The treatment regimen for DEXii should be the PRN with bi-monthly or quarterly monitoring visits (with a safety visit at 6-8 weeks after DEXii for IOP control). After injecting FAc patient should be monitored at least quarterly to rule out possible side effects. If a patient does not respond to corticosteroids, switch again to anti-VEGF therapy, combine therapies or reevaluate patients diagnose (Figure 1).

\section{Conclusion}

There are significant unmet needs in the current management of DME. With a significant proportion of patients not responding to the first-line anti-VEGF therapy, it is important to identify non-responders through inflammatory biomarkers to provide the most efficacy therapy for each patient in individualized treatment. ${ }^{34}$ We have tried to bring together the scientific knowledge from randomized clinical trials, with real-world studies and our clinical experience. The management of DME requires the work of a multidisciplinary team to achieve treatment optimization and maximizing outcomes.
Several algorithms and guidelines have been proposed for the treatment of this multifactorial disease to improve patients' vision and quality of life for all involved. Figure 1 highlights our recommendation for an optimized treatment. There is a resilient need to understand the disease, its treatments, regimens available and convenience for all involved to propose an adequate algorithm for the treatment of DR and DME in an individualized regimen.

Despite the emergence of consensus guidelines as well as algorithms, DR and DME remain challenging to treat.

Innovative therapies approaches are in the pipeline to improve compliance, extending the duration of action and thus reducing the need for frequent injections and to improve optimization of the functional and anatomical, such as the port delivery systems with ranibizumab (Genentech/Roche, Phase III, VEGF-A antagonist), brolucizumab (Novartis, Phase III, VEGF-A antagonist), faricimab (Genentech/Roche, Phase III, VEGF-A antagonist), KSI-301 (Kodiak Sciences Inc., Phase III, VEGF antagonists), MYL-1701P (Momenta Pharmaceuticals/Mylan, Phase III, VEGF-A antagonist) and Gene Therapy among others. $^{56-61}$

Further understanding of the contributing factors to the development and progression of DR should bring new drug discoveries for more effective and better-tolerated treatments.

\section{Acknowledgments}

Medical writing assistance was provided by Manuscriptedit.

\section{Author Contributions}

All authors made a significant contribution to the work reported, whether that is in the conception, study design, execution, acquisition of data, analysis and interpretation, or in all these areas; took part in drafting, revising or critically reviewing the article; gave final approval of the version to be published; have agreed on the journal to which the article has been submitted; and agree to be accountable for all aspects of the work.

\section{Funding}

Allergan plc provided financial support for the work conducted to prepare this manuscript but was not involved directly in preparation of the manuscript or the decision to publish. 


\section{Disclosure}

Figueira $\mathrm{J}$ contribute in consultancy or advisory roles from Alcon, Allergan, Alimera, Bayer, Novartis and Roche. Henriques $\mathrm{J}$ contribute in consultancy or advisory roles from Alcon, Allergan, Alimera, Bayer, Roche and Novartis. Carneiro A contribute in consultancy or advisory roles from Allergan, Alimera, Bayer, Novartis and Roche. Neves $\mathrm{C}$ contribute in consultancy or advisory roles from Allergan, Bayer and Flores R contribute in consultancy or advisory roles from Allergan, Bayer, Novartis and Roche. CastroSousa JP contribute in consultancy or advisory roles from Allergan, Bayer, Novartis and Roche. Meireles A contribute in consultancy or advisory roles from Alcon, Allergan, Alimera and Novartis. Gomes $\mathrm{N}$ contribute in consultancy or advisory roles from Allergan, Bayer and Novartis. Nascimento $\mathbf{J}$ contribute in consultancy or advisory roles from Allergan, Bayer and Novartis. Amaro $M$ contribute in consultancy or advisory roles from Allergan, Bayer, Novartis and Zeiss. Silva R is a member of advisory board for Allergan, Alimera, Bayer, Novartis, NovoNordisk, Thea and Roche. The authors report no other conflicts of interest in this work.

\section{References}

1. Romero-Aroca $\mathrm{P}$, Fernández-Balart $\mathrm{J}$, Baget-Bernaldiz $\mathrm{M}$, et al. Changes in the diabetic retinopathy epidemiology after 14 years in a population of Type 1 and 2 diabetic patients after the new diabetes mellitus diagnosis criteria and a more strict control of the patients. $J$ Diabetes Complications. 2009;23(4):229-238. doi:10.1016/j. jdiacomp.2008.02.012

2. Romero-Aroca P. Targeting the pathophysiology of diabetic macular edema. Diabetes Care. 2010;33(11):2484-2485. doi:10.2337/dc101580

3. American Diabetes Association. 6. Glycemic targets: standards of medical care in diabetes-2019. Diabetes Care. 2019;42(Suppl 1): S61-S70. doi:10.2337/dc19-S006

4. Schmidt-Erfurth U, Garcia-Arumi J, Bandello F, et al. Guidelines for the management of diabetic macular edema by the European Society of Retina Specialists (EURETINA). Ophthalmologica. 2017;237 (4):185-222. doi:10.1159/000458539

5. The Diabetic Retinopathy Study Research Group. Photocoagulation treatment of proliferative diabetic retinopathy. Clinical application of Diabetic Retinopathy Study (DRS) findings, DRS report number 8. Ophthalmol. 1981;88(7):583-600.

6. Jampol L, Bressler N, Glassman A. Revolution to a new standard treatment of diabetic macular edema. JAMA. 2014;311 (22):2269-2270. doi:10.1001/jama.2014.2536

7. Figueira J, Khan J, Nunes S, et al. Prospective randomised controlled trial comparing sub-threshold micropulse diode laser photocoagulation and conventional green laser for clinically significant diabetic macular oedema. Br J Ophthalmol. 2009;93(10):1341-1344. doi:10.1136/ bjo.2008.146712

8. Vujosevic S, Martini F, Longhin E, Convento E, Cavarzeran F, Midena E. Subthreshold micropulse yellow laser versus subthreshold micropulse infrared laser in center-involving diabetic macular edema: morphologic and functional safety. Retina. 2015;35(8):1594-1603. doi:10.1097/IAE.0000000000000521
9. Vujosevic S, Bottega E, Casciano M, Pilotto E, Convento E, Midena E. Microperimetry and fundus autofluorescence in diabetic macular edema: subthreshold micropulse diode laser versus modified early treatment diabetic retinopathy study laser photocoagulation. Retina. 2010;30(6):908-916. doi:10.1097/IAE.0b013e3181c96986

10. Scholz P, Altay L, Fauser S. A review of subthreshold micropulse laser for treatment of macular disorders. Adv Ther. 2017;17 (34):1528-1555. doi:10.1007/s12325-017-0559-y

11. Gawęcki M. Micropulse laser treatment of retinal diseases. $J$ Clin Med. 2019;8(2):242. doi:10.3390/jcm8020242

12. Moshfeghi DM, Kaiser PK, Michels S, et al. The role of anti-VEGF therapy in the treatment of diabetic macular edema. Ophthalmic Surg Lasers Imaging Retina. 2016;47(6 Suppl):S4-S14. doi:10.3928/ 23258160-20160415-01

13. Sarwar S, Bakbak B, Sadiq MA, et al. Fusion proteins: aflibercept (VEGF Trap-Eye). Dev Ophthalmol. 2016;55:282-294.

14. Ferrara N, Adamis AP. Ten years of anti-vascular endothelial growth factor therapy. Nat Rev Drug Discov. 2016;15(6):385-403.

15. Cai S, Bressler NM. Aflibercept, bevacizumab or ranibizumab for diabetic macular oedema: recent clinically relevant findings from DRCR.net protocol T. Curr Opin Ophthalmol. 2017;28(6):636-643. doi:10.1097/ICU.0000000000000424

16. Chawan-Saad J, Wu M, Wu A, Wu L. Corticosteroids for diabetic macular edema. Taiwan $J$ Ophthalmol. 2019;9(4):233-242. doi:10.4103/tjo.tjo_68_19

17. Urias EA, Urias GA, Monickaraj F, McGuire P, Das A. Novel therapeutic targets in diabetic macular edema: beyond VEGF. Vision Res. 2017;139:221-227. doi:10.1016/j.visres.2017.06.015

18. Haller JA, Kuppermann BD, Blumenkranz MS, et al. Randomized controlled trial of an intravitreous dexamethasone drug delivery system in patients with diabetic macular edema. Arch Ophthalmol. 2010;128(3):289-296. doi:10.1001/archophthalmol.2010.21

19. Campochiaro PA, Hafiz G, Shah SM, et al. Sustained ocular delivery of fluocinolone acetonide by an intravitreal insert. Ophthalmology. 2010;117(7):1393-1399.e3. doi:10.1016/j.ophtha.2009.11.024

20. Bressler SB, Glassman AR, Almukhtar T, et al. Five-year outcomes of ranibizumab with prompt or deferred laser versus laser or triamcinolone plus deferred ranibizumab for diabetic macular edema. $\mathrm{Am}$ J Ophthalmol. 2016;164:57-68. doi:10.1016/j.ajo.2015.12.025

21. Fung AT, Tran T, Lim LL, et al. Local delivery of corticosteroids in clinical ophthalmology: a review. Clin Exp Ophthalmol. 2020;48 (3):366-401.

22. Heier JS, Korobelnik JF, Brown DM. Intravitreal aflibercept for diabetic macular edema: 148-week results from the VISTA and VIVID studies. Ophthalmology. 2016;123(11):2376-2385. doi:10.1016/j.ophtha.2016.07.032

23. Prünte C, Fajnkuchen F, Mahmood S. Ranibizumab 0.5mg treat-andextend regimen for diabetic macular oedema: the RETAIN study. Brit $J$ Ophthalmol. 2016;100(6):787-795. doi:10.1136/bjophthalmol2015-307249

24. Aiello LP, Avery RL, Arrigg PG, et al. Vascular endothelial growth factor in ocular fluid of patients with diabetic retinopathy and other retinal disorders. New Engl J Med. 1994;331(22):1480-1487. doi:10.1056/NEJM199412013312203

25. Fong DS, Strauber SF, Aiello LP. Comparison of the modified early treatment diabetic retinopathy study and mild macular grid laser photocoagulation strategies for diabetic macular edema. Arch Ophthalmol. 2007;125(4):469-480.

26. Lavinsky D, Sramek C, Wang J. Subvisible retinal laser therapy: titration algorithm and tissue response. Retina. 2014;34(1):87-97. doi:10.1097/IAE.0b013e3182993edc

27. Avery RL, Gordon GM. Systemic safety of prolonged monthly antivascular endothelial growth factor therapy for diabetic macular edema: a systematic review and meta-analysis. JAMA Ophthalmol. 2016;134(1):21-29. doi:10.1001/jamaophthalmol.2015.4070 
28. Chandra S, Sheth J, Anantharaman G, Gopalakrishnan M. Ranibizumab-induced retinal reperfusion and regression of neovascularization in diabetic retinopathy: an angiographic illustration. Am J Ophthalmol Case Rep. 2018;9:41-44. doi:10.1016/j.ajoc.2018.01.006

29. Medeiros MD, Alkabes M, Nucci P. Effectiveness of the dexamethasone intravitreal implant for treatment of patients with diabetic macular oedema. Eur Endocrinol. 2014;10(2):111. doi:10.17925/ EE.2014.10.02.111

30. Boyer DS, Faber D, Gupta S, et al. Dexamethasone intravitreal implant for treatment of diabetic macular edema in vitrectomized patients. Retina. 2011;31(5):915-923. doi:10.1097/ IAE.0b013e318206d18c

31. Hostovsky A, Muni RH, Eng KT, Mulhall D, Leung C, Kertes PJ. Intraoperative dexamethasone intravitreal implant (ozurdex) in vitrectomy surgery for epiretinal membrane. Curr Eye Res. 2020;45 (6):737-741. doi:10.1080/02713683.2019.1697454

32. Chang YC, Liu PK, Kao TE, et al. Dexamethasone intravitreal implant (ozurdex) for long-term macular edema after epiretinal membrane peeling surgery. J Ophthalmol. 2018;2018:5832186.

33. García-Layana A, Figueroa MS, Arias L, et al. Clinical decision-making when treating diabetic macular edema patients with dexamethasone intravitreal implants. Ophthalmologica. 2018;240(2):61-72. doi:10.1159/000486800

34. Kodjikian L, Bellocq D, Bandello F, et al. First-line treatment algorithm and guidelines in center-involving diabetic macular edema. Eur J Ophthalmol. 2019;29(6):573-584. doi:10.1177/ 1120672119857511

35. Vujosevic S, Simó R. Local and systemic inflammatory biomarkers of diabetic retinopathy: an integrative approach. Invest Ophthalmol Vis Sci. 2017;58(6):BIO68-BIO75. doi:10.1167/iovs.17-21769

36. Vujosevic S, Torresin T, Bini S. Imaging retinal inflammatory biomarkers after intravitreal steroid and anti-VEGF treatment in diabetic macular oedema. Acta Ophthalmol. 2017;95(5):464-471. doi:10.1111/aos.13294

37. Santos AR, Costa MÂ, Schwartz C. Optical coherence tomography baseline predictors for initial best-corrected visual acuity response to intravitreal anti-vascular endothelial growth factor treatment in eyes with diabetic macular edema: the CHARTRES Study. Retina. 2018;38(6):1110-1119. doi:10.1097/IAE.0000000000001687

38. Gerendas BS, Prager S, Deak G. Predictive imaging biomarkers relevant for functional and anatomical outcomes during ranibizumab therapy of diabetic macular oedema. Br J Ophthalmol. 2018;102 (2):195-203. doi:10.1136/bjophthalmol-2017-310483

39. Zur D, Iglicki M, Busch C, et al. OCT biomarkers as functional outcome predictors in diabetic macular edema treated with dexamethasone implant. Ophthalmology. 2018;125(2):267-275. doi:10.1016/j.ophtha.2017.08.031

40. Meduri A, Oliverio GW, Trombetta L, et al. Optical coherence tomography predictors of favorable functional response in naïve diabetic macular edema eyes treated with dexamethasone implants as a first-line agent. J Ophthalmol. 2021;2021:6639418. doi:10.1155/2021/6639418

41. Campochiaro PA, Brown DM, Pearson A, et al. Sustained delivery fluocinolone acetonide vitreous inserts provide benefit for at least 3 years in patients with diabetic macular edema. Ophthalmology. 2012;119(10):2125-2132. doi:10.1016/j.ophtha.2012.04.030

42. Bilgic A, Aditya S, Laurent K, et al. Pro re nata dexamethasone implant for treatment-naive phakic eyes with diabetic macular edema: a prospective study. Ophthalmol Ret. 2019;3(11):929-937. doi:10.1016/j.oret.2019.05.027

43. Malcles A, Janin-Manificat H, Yhuel Y, et al. Anterior chamber migration of intravitreal dexamethasone implant $\left(\right.$ Ozurdex $\left.{ }^{\circledR}\right)$ in pseudophakic eyes: report of three cases. J Francaisd' Ophtalmologie. 2013;36(4):327-362.
44. Early Treatment Diabetic Retinopathy Study Research Group. Grading diabetic retinopathy from stereoscopic color fundus photographs-an extension of the modified Airlie House classification. ETDRS report number 10. Ophthalmology. 1991;98(5):786-806. doi:10.1016/S0161-6420(13)38012-9

45. Manousaridis K, Talks J. Macular ischaemia: a contraindication for anti-VEGF treatment in retinal vascular disease? Br J Ophthalmol. 2012;96(2):179-184. doi:10.1136/bjophthalmol-2011-301087

46. Castro-Navarro V, Cervera-Taulet E, Navarro-Palop C, et al. Intravitreal dexamethasone implant Ozurdex ${ }^{\circledR}$ in naïve and refractory patients with different subtypes of diabetic macular edema. BMC Ophthalmol. 2019;19(1):15. doi:10.1186/s12886018-1022-9

47. Scott G, Ivor J, Gregory L, et al. Diabetes and cardiovascular disease: a statement for healthcare professionals from the American Heart Association. Circulation. 1999;100(10):1134-1146. doi:10.1161/01. CIR.100.10.1134

48. Avery RL, Castellarin AA, Steinle NC. Systemic pharmacokinetics and pharmacodynamics of intravitreal aflibercept, bevacizumab, and ranibizumab. Retina. 2017;37(10):1847-1858. doi:10.1097/ IAE.0000000000001493

49. Heier JS, Bressler NM, Avery RL. Comparison of aflibercept, bevacizumab, and ranibizumab for treatment of diabetic macular edema: extrapolation of data to clinical practice. JAMA Ophthalmol. 2016;134(1):95-99. doi:10.1001/jamaophthalmol.2015.4110

50. Polizzi S, Mahajan VB. Intravitreal anti-VEGF injections in pregnancy: case series and review of literature. J Ocular Pharmacol Therap. 2015;31(10):605-610. doi:10.1089/jop.2015.0056

51. Yoo R, Kim HC, Chung H. Dexamethasone intravitreal implant for diabetic macular edema in a pregnant patient. Int $J$ Ophthalmol. 2016;9(10):1524.

52. Rosenthal JM, Johnson MW. Management of retinal diseases in pregnant patients. J Ophthalmic Vis Res. 2018;13(1):62-65. doi:10.4103/jovr.jovr_195_17

53. Ehlken C, Helms M, Böhringer D, Agostini HT, Stahl A. Association of treatment adherence with real-life VA outcomes in AMD, DME, and BRVO patients. Clin Ophthalmol. 2018;12:13-20. doi:10.2147/ OPTH.S151611

54. Wubben TJ, Johnson MW; Anti-VEGF treatment interruption study group. Anti-vascular endothelial growth factor therapy for diabetic retinopathy: consequences of inadvertent treatment interruptions. Am J Ophthalmol. 2019;204:13-18. doi:10.1016/j. ajo.2019.03.005

55. Busch C, Fraser-Bell S, Iglicki M, et al. Real-world outcomes of non-responding diabetic macular edema treated with continued anti-VEGF therapy versus early switch to dexamethasone implant: 2-year results. Acta Diabetol. 2019;56(12):1341. doi:10.1007/ s00592-019-01416-4

56. Roche. Efficacy, safety, and pharmacokinetics of the port delivery system with ranibizumab in participants with diabetic macular edema compared with intravitreal ranibizumab (Pagoda). Available from: https:/clinicaltrials.gov/ct2/show/NCT04108156. NLM identifier: NCT04108156. Accessed May 31, 2021.

57. Novartis. A study of the efficacy and safety of brolucizumab vs. aflibercept in patients with visual impairment due to diabetic macular edema (KITE). Available from: https:/clinicaltrials.gov/ct2/show/ NCT03481660. NLM identifier: NCT04079231. Accessed May 31, 2021.

58. Roche. A study to evaluate the efficacy and safety of faricimab (RO6867461) in participants with diabetic macular edema (YOSEMITE). Available from: https://clinicaltrials.gov/ct2/show/ NCT03622580. NLM identifier: NCT03622580. Accessed May 31, 2021. 
59. Kodiac Sciences Inc. A Study to Evaluate the Efficacy, Durability, and Safety of KSI-301 Compared to Aflibercept in Participants With Diabetic Macular Edema (DME) (GLIMMER). Available from: https://clinicaltrials.gov/ct2/show/NCT04603937. NLM identifier: NCT04603937. Accessed May 31, 2021.

60. Mylan Inc. Comparative study to evaluate the efficacy and safety of MYL-1701P and Eylea ${ }^{\circledR}$ in subjects with diabetic macular edema. Available from: https://clinicaltrials.gov/ct2/show/NCT03610646. NLM identifier: NCT03610646. Accessed May 31, 2021.
61. Adverum Biotechnologies, Inc. ADVM-022 Intravitreal Gene Therapy for DME (INFINITY). Available from: https://clinicaltrials. gov/ct2/show/NCT04418427. NLM identifier:NCT044184276. Accessed May 31, 2021.

\section{Publish your work in this journal}

Clinical Ophthalmology is an international, peer-reviewed journal covering all subspecialties within ophthalmology. Key topics include: Optometry; Visual science; Pharmacology and drug therapy in eye diseases; Basic Sciences; Primary and Secondary eye care; Patient Safety and Quality of Care Improvements. This journal is indexed on PubMed

Submit your manuscript here: https://www.dovepress.com/clinical-ophthalmology-journal
Central and CAS, and is the official journal of The Society of Clinical Ophthalmology (SCO). The manuscript management system is completely online and includes a very quick and fair peer-review system, which is all easy to use. Visit http://www.dovepress.com/ testimonials.php to read real quotes from published authors. 